\title{
Management of dementia-related agitation: between the devil and the deep blue sea
}

\author{
Michael J Passmore
}

Department of Psychiatry, University of British Columbia, Mount St. Joseph Hospital, Ward 1 South, 3080 Prince Edward St, Vancouver, British Columbia, Canada V5T 3N4; passmoremd@gmail.com

\section{WHAT IS ALREADY KNOWN ON THIS TOPIC?}

Dementia-related agitation is a common and costly condition that is distressing, debilitating, often dangerous and usually difficult to treat in a safe and reliable manner. ${ }^{1}$ Clinicians are caught between the 'devil' of medications with unfavourable risk-benefit profiles $^{2}$ and the 'deep blue sea' of non-pharmacological interventions perceived as impractical and lacking in evidence for benefit. ${ }^{3}$ Before opting for any intervention, it is essential to rule out underlying causes of agitation and then consider strategies such as environmental modification, sensory interventions and family involvement/support.

\section{WHAT DOES THIS PAPER ADD?}

- In care-home dementia residents, training paid caregivers in personcentred care, communication skills and dementia mapping (focusing on patient needs and with supervision of trained caregivers during implementation) significantly reduced agitation at implementation and for up to 6 months afterwards.

- Group activities, sensory intervention and music therapy all prevented agitation during the intervention; sensory interventions also reduced clinically significant symptoms of agitation.

- Aromatherapy, light therapy and home-like care do not reduce agitation.

\section{LIMITATIONS}

- The complex and dynamic nature of dementia-related agitation, along with the subjective nature of many non-pharmacological interventions, make it a very challenging syndrome to study in a rigorously controlled manner.

- Similar to a placebo effect, the Hawthorne effect, that is, the tendency for participants to improve simply by virtue of involvement in the control arm of non-pharmacological intervention studies requires further consideration as a potential confounding factor.

\section{WHAT NEXT IN RESEARCH?}

To evaluate the implementation of promising non-pharmacological management strategies in different dementia care settings and to manualise and disseminate pragmatic strategies in order to foster positive changes in dementia care culture.

\section{DO THESE RESULTS CHANGE YOUR PRACTICES AND WHY?}

The results of this review will encourage me to focus on providing support and training for caregivers of older adults with dementia. It will also be important to have ongoing collaboration with nursing leaders and facility administrators to ensure a focus on specific factors such as supervision of caregivers-in-training, as well as incorporation of sensory interventions into dementia care plans. As the authors point out, although few level 1 evidence-based studies existed for inclusion in their review, a relative lack of evidence is not evidence for lack of efficacy, therefore non-pharmacological interventions should remain first-line prevention and treatment strategies for dementia-related agitation.

Competing interests None.

doi:10.1136/eb-2014-101931

\section{REFERENCES}

1. Kales HC, Gitlin LN, Lyketsos CG, et al. Management of neuropsychiatric symptoms of dementia in clinical settings: recommendations from a multidisciplinary expert panel. J Am Geriatr Soc 2014;62:762-9.

2. Naarding $\mathbf{P}$, van Grevenstein M, Beekman AT. Benefit-risk analysis for the clinician: 'primum non nocere' revisited-the case for antipsychotics in the treatment of behavioural disturbances in dementia. Int J Geriatr Psychiatry 2010;25:437-40.

3. Kong EH, Evans LK, Guevara JP. Nonpharmacological intervention for agitation in dementia: a systematic review and meta-analysis. Aging Ment Health 2009;13:512-20.

ABSTRACT FROM: Livingston G, Kelly L, Lewis-Holmes E, et al. A systematic review of the clinical effectiveness and cost-effectiveness of sensory, psychological and behavioural interventions for managing agitation in older adults with dementia. Health Technol Assess 2014;18:1-226.

Data sources Web of Knowledge, EMBASE, British Nursing Index, the HTA programme database, PsycINFO, NHS Evidence, System for Information on Grey Literature, CINAHL and The Cochrane Library were searched between August 2011 and June 2012. Reference lists were handsearched and authors contacted.

Study type included RCTs, cohort and observational studies investigating non-pharmacological interventions for agitation in adults aged over 50 years with any type of dementia in any setting.

Intervention Psychological, behavioural, environmental and sensory interventions. This included dementia care mapping (where a supervisor watches an individual with dementia and feeds back to the carer which factors improve the person's well-being and what are the potential environmental triggers, so that changes can be made to the care plan).

Comparison Either a separate control group or before and after studies in the same participants.

\section{OUTCOMES}

Study characteristics One hundred and sixty studies (97 high-quality RCTs) were identified, ranging from 4 to 387 participants. Most studies were conducted in care homes, 77 were conducted in the USA, 13 in Australia, 13 in the UK, 10 in Canada, and the remaining in Europe, South Korea, Japan, China, Iran, Iceland and Israel. Meta-analysis was not possible due to the heterogeneity of the studies.
Interventions with evidence of efficacy In care home residents, activities and music therapy using a specific protocol reduced agitation levels in the immediate term (standardised effect sizes (SES) -0.8 to -0.6 for activities; SES -0.8 to -0.5 for music therapy) but there was no evidence of sustained effects, or effect on severe agitation. Sensory interventions (including massage, therapeutic touch and multisensory stimulation) were also effective in reducing mean and clinically significant agitation (SES -1.3 to -0.6 ) but there was no evidence of longer term effects. Training and supervising paid caregivers in communication or personcentred skills immediately improved severe agitation with effects lasting up to 6 months in care homes and supported living residences (SES -1.8 to -0.4$)$. Dementia care mapping with supervision was immediately effective and reduced agitation over 4 months (SES -1.4 to -0.3 ).

Interventions with no evidence of efficacy: Light therapy (30-60 min of daily bright-light exposure), home-like care (homes set up like a domestic environment with eight or less residents and meals prepared by a small fixed staff team and residents or carers) and aromatherapy.

Interventions with too little evidence to make definitive recommendations: Training family caregivers in behavioural management, training family caregivers in cognitive-behavioural therapy, working with the person with dementia in a care home, music therapy not following a specific proto$\mathrm{col}$, exercise, pet therapy, changing the environment, and working through paid caregivers in care homes without supervision. 